\title{
A Robust Watermarking Scheme for City Image
}

\author{
Ruichen Jin ${ }^{1}$ and Jongweon $\mathrm{Kim}^{2 *}$ \\ ${ }^{1}$ Department of Copyright Protection, Sangmyung University, Seoul, Korea \\ ${ }^{2}$ Department of Contents and Copyright, Sangmyung University, Seoul, Korea \\ 1jinruichen@cclabs.kr, ${ }^{2}$ jwkim@smu.ac.kr
}

\begin{abstract}
In this paper, we propose an effective watermarking scheme based on Radon transform for city image. The robustness against rotation attack is achieved using the translation property of the Radon transform. The Radon transform emphasizes and detects the linear characteristic to calculate the angle of image rotation. The watermark is a hologram generated by quantization based on the cover image. We used hologram quantization to spread the watermark information and analyze the cover image detail. The hologram is transformed by a discrete fractional random transform (DFRNT) with a random seed $\beta$. It makes the watermark security. The proposed method uses discrete wavelet transform (DWT) domain. DWT domain watermarking is robust to signal processing attacks. For improving the accuracy of detecting, we fix the center of image and swivel that the pixels of image boundary move 1 notch at a time. The proposed algorithm also robust to the spatial Effect attack. We have performed an intensive simulation to show the robustness in rotation of the proposed method.
\end{abstract}

Keywords: rotation invariant, image, digital watermarking, radon transform, hologram, discrete wavelet transform, discrete fractional random transform, image normalization

\section{Introduction}

As increased interest in intellectual property rights, the demand for the copyright protection technology is getting a lot. With the development of science and copyright protection technology, many high-performance multimedia devices are produced and it produce high definition multimedia products. Thus we have to develop a corresponding technique.

Digital watermarking is an efficient solution for copyright protection, which inserts copyright information into the contents [1-4]. In order to protect the information effectively, the watermarking methods should be robust to various attacks. Geometric attack, such as rotation, scaling and translation (RST) is known as one of the most difficult attacks to resist. There are lots researches resistant to geometric attacks. The existing schemes can be classified into: non-blind scheme [5], invariant domain embedding [6, 7], template based synchronization [8, 9] and feature-based synchronization [10-18].

In the industry of film and drama, the location hunting is the important part of whole process. Even recently, a new job as location manager is beginning to come up. In this paper, we focus on the need for protecting the copyright of city image.

This paper proceeds as follow. We shall first briefly introduce related concepts for watermarking schemes in section 2. Such as hologram, DFRNT, Radon transform and edge detection. In section 3, details on the image normalization is discussed in later sections and show the watermark embedding/extracting process. In section 4 , we show the

Jongweon Kim Corresponding Author. 
show the watermark embedding/extracting Experiments and the values of extracting watermark as Results.

\section{Related Works}

\subsection{Holograms and DFRNT}

Holograms were first proposed by Gabor in 1948 [19], and coherent light interference holograms, which use two separate off-axis holograms, were released by Leith and Upatnieks in 1962 [20]. Holograms employ a technique that allows the light scattered from an object to be recorded and later reconstructed, so that when an imaging system is placed in the reconstructed beam, an image of the object will be seen even when the object is no longer present. The image changes with the position and orientation of the viewing system in exactly the same way as if the object were still present, thus making the image appear three-dimensional. The hologram recoding itself is not an image; it consists of an apparently random structure of varying intensity, density or profile.

The object wave and reference wave equation using Fourier holograms is as follows:

$$
U_{H}(\xi, \eta)=I(\xi, \eta)^{*} R(\xi, \eta)+I(\xi, \eta) R(\xi, \eta)^{*}
$$

To restore the information representing the complex amplitude of the wave in Equation (1), we use the following expression:

$$
U_{R}(\xi, \eta)=R(\xi, \eta) \exp \left\{-j \frac{2 \pi}{\lambda_{2} f^{\prime}}\left(\xi x_{r}^{\prime}+\eta y_{r}^{\prime}\right)\right\}
$$
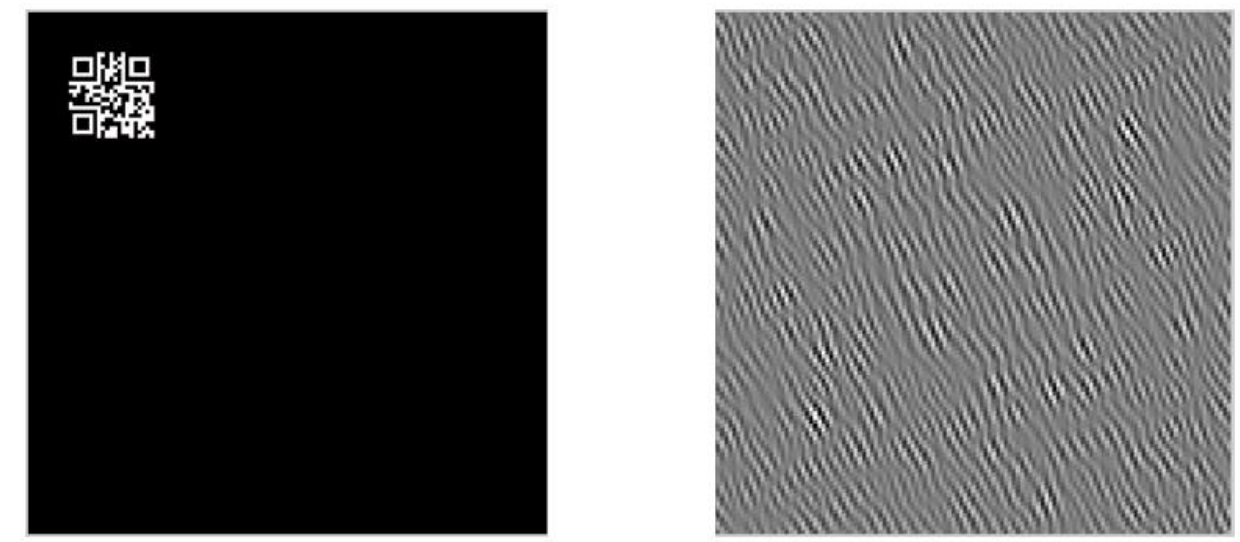

Figure 1. Hologram Generation of a QR code

In this paper, we use a $\mathrm{QR}$ code as the watermark information. QR codes contain of square dots (black modules) arranged in a square pattern on a white background. They have become popular and many people used in a range of environments because of their fast readability and high data capacity. [21]

Using hologram quantization, we can restore the QR code effectively. If a hologram (N $\times_{N}$ ) is generated using a $\mathrm{QR}$ code, then the range of the value which average of wavelet coefficient is $(-n, n)$. According to observations, a minimal portion of values approach the extremes of this range, whereas above $98 \%$ of them are located in $(-n / 2, n / 2)$. We can appoint a threshold range so that values outside the range are changed to the closest value in the quantization threshold range. Figure 2.-(a) shows the histogram of hologram values. Following quantization, the hologram values are distributed as in Figure 2.-(b). 


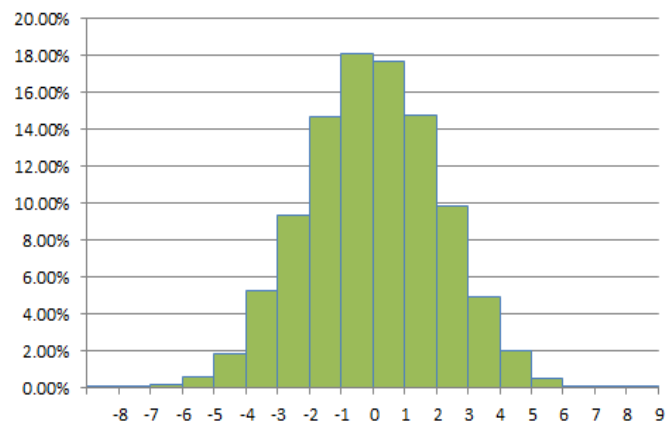

(a)

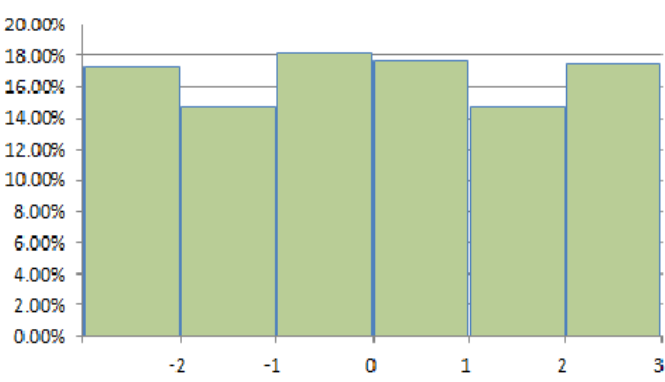

(b)

Figure 2. Histograms of Hologram Values before and after Quantization

A quantization table is formed using a random integer matrix of values from 0 to $\mathrm{m}$. Each $\mathrm{M} \times \mathrm{M}$ block of the changed hologram is matched to the relevant integer in the quantization table, and is then multiplied by this value.

The discrete fractional Fourier transform (DFRNT) is defined by Zeev Zalevsky in [22]. The randomness is generated by a random matrix. The overall process is quite similar to that by which the transform matrix of the DFRNT is obtained. The DFRNT can be defined by a diagonal symmetric random matrix. Also it is linear, unitary, indexadditive and energy conserving. However, its kernel transform matrix is random, and this affords high security in information security applications such as digital watermarking.

\subsection{Radon Transform}

The Radon transform is the integral transform consisting of the integral of a function over straight lines. The transform was introduced in 1917 by Radon [23]. It is widely applicable to tomography for the creation of an image from the projection data associated with cross-sectional scans of an object. The Radon transform of image function $f(x, y)$ is denoted by $\mathrm{R}(\theta, \mathrm{r})$, which is defined as follows;

$\mathrm{R}(\theta, \mathrm{r})=\iint f(x, y) \delta(r-x \cos \theta-y \sin \theta) d x d y$

Where $\delta$ is the Dirac function. Where $\theta \in[0, \pi)$ denoting the angle between the beam and $\mathrm{x}$-axis. $\mathrm{r} \in(-\infty, \infty)$ is the perpendicular distance from the beam crossing the origin. The Figure 3. shows the corresponding discrete Radon domain with lena image. 

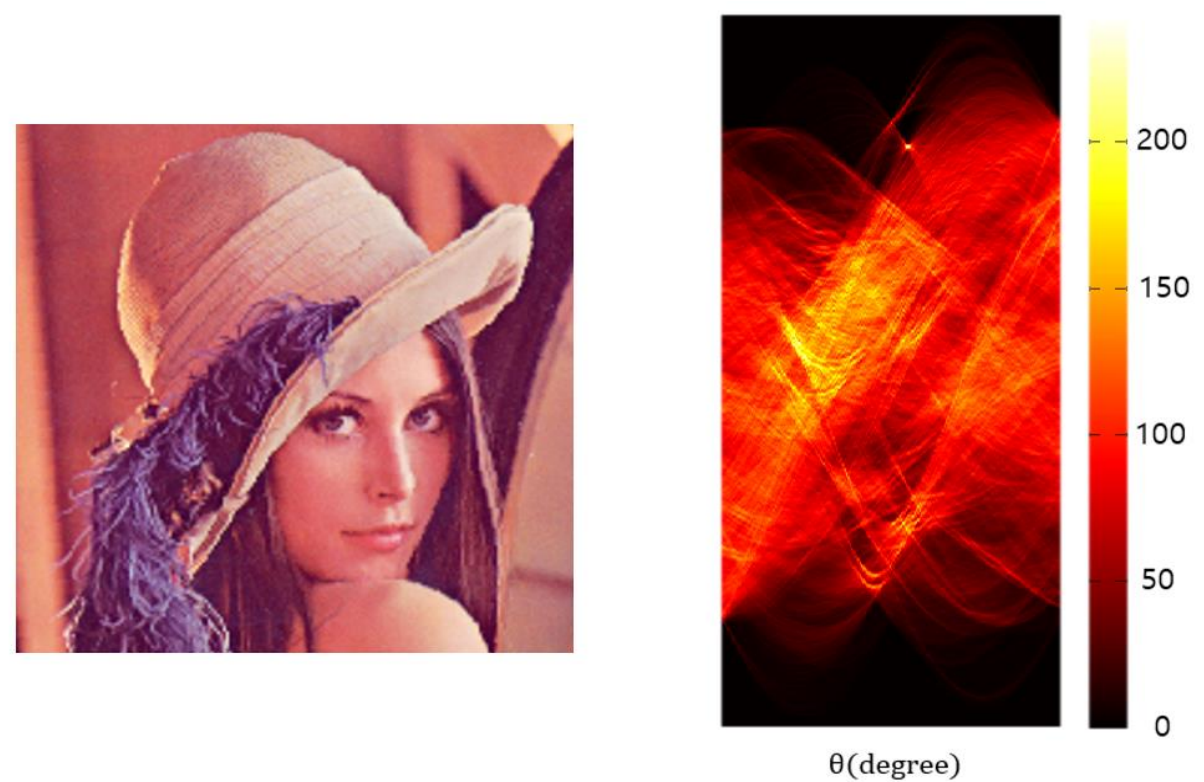

Figure 3. The Corresponding Discrete Radon Domain

\subsection{Edge Detection}

For detecting the edge of an image, we must find the boundaries of the area. It is the brightness changes rapidly taking place in image. It offers important clues in the recognition with easy calculation.

Several algorithms exists, and in this paper we are focusing on a particular one developed by John F. Canny (JFC) in 1986. Even though it is quite old, it has become one of the standard edge detection methods and it is still used in research.

The Process of Canny edge detection algorithm can be broken down to 5 different steps:

1. Apply Gaussian filter to smooth the image in order to remove the noise.

2. Find the intensity gradients of the image.

3. Apply non-maximum suppression to get rid of spurious response to edge detection.

4. Apply double threshold to determine potential edges.

5. Track edge by hysteresis: Finalize the detection of edges by suppressing all the other edges that are weak and not connected to strong edges.

Figure 4. shows the edge detection using Canny edge detection algorithm.
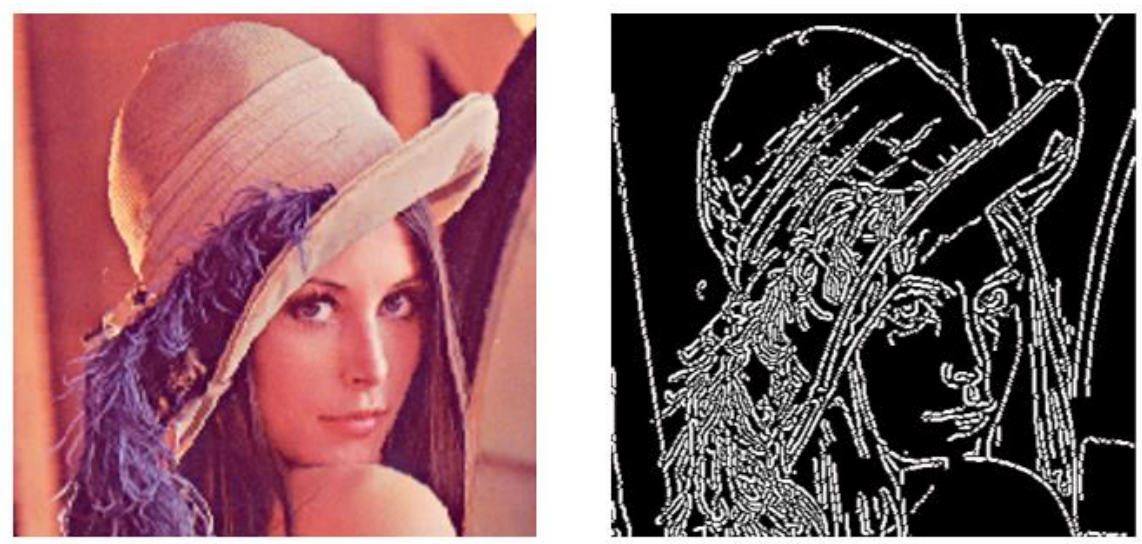

Figure 4. Canny Edge Detection 


\section{Proposed Algorithm}

\subsection{Image Normalization}

Our scheme uses image normalization resistant against rotation attack. Figure 5. shows the process of image normalization. Through the image normalization, rotated image can be corrected.

Step 1: Detect the edge of the original image using canny detect operator.

Step 2: Linking the edge with thickening operation.

Step 3: Calculate the rotation angle and normalize image.

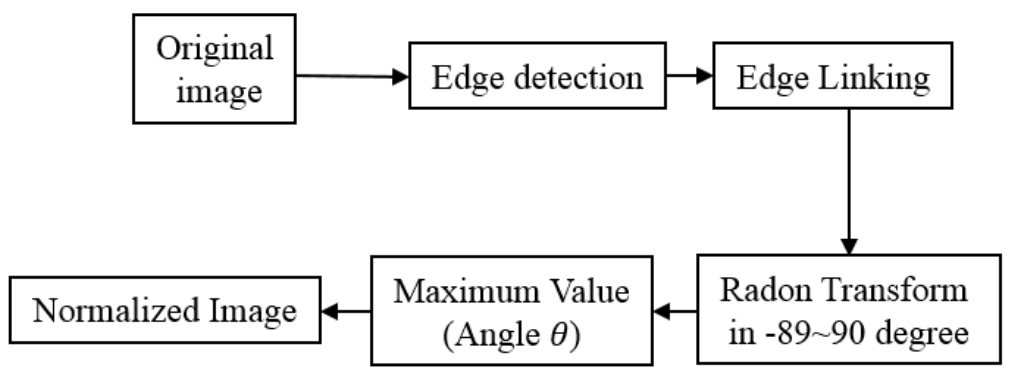

Figure 5. Image Normalization Process

\subsection{Embedding Scheme}

Figure 6. shows the process of the embedding scheme.

Step 1: Encoding the watermark message by QR code encoder.

Step 2: Transform the QR code using a DFRNT with seed $\beta$, and generate the hologram.

Step 3: Normalize the original city image and get angle value by radon transform.

Step 4: Rotate the hologram by angle $\theta$. And get the new matrix.

Step 5: Transform them by two-depth, two- dimension Inverse DWT.

Step 6: Add original images and Step 5.

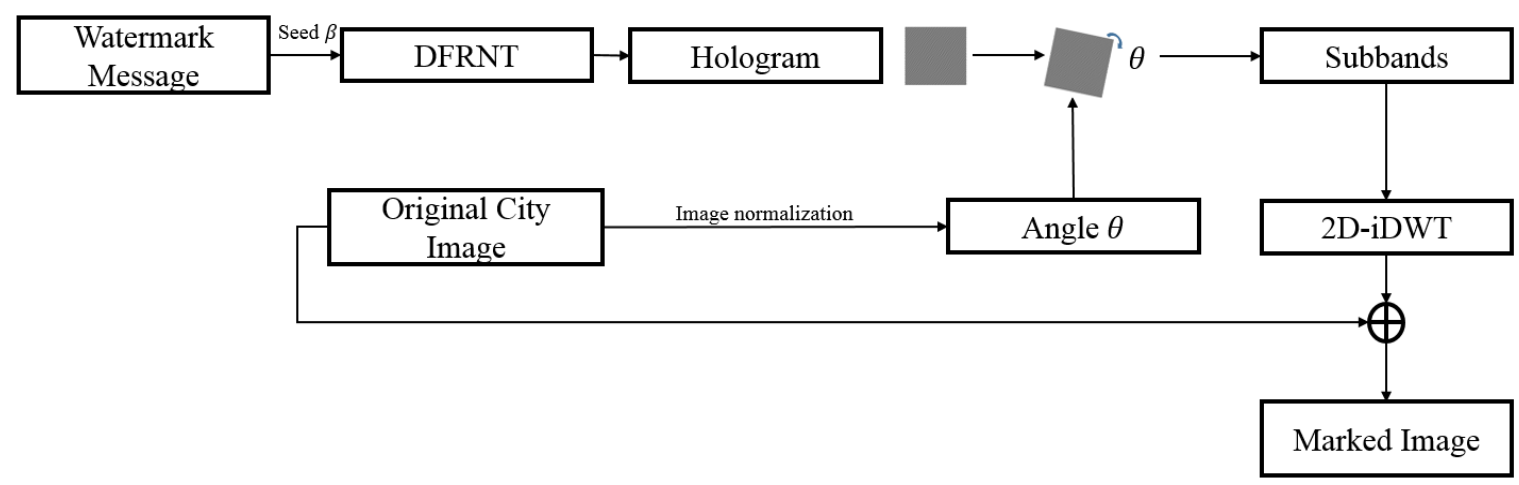

Figure 6. Watermark Embedding Process 


\subsection{Extraction Scheme}

The extraction process is the reverse of the embedding process, and uses the same seed $\beta$ in the DFRNT. Figure 7. shows the process of the extraction scheme.

Step 1: Normalize the marked image.

Step 2: Transform the matrix using a two-depth, two- dimension DWT, and select the subbands.

Step 3: Add the subbands and transform them by DFRNT with seed $\beta$.

Step 4: Restore them with ReHologram, and decode with QR decoder.

Step 5: If the QR decoder cannot decode the message, then distort the image. Loop the step2 to step5, until the decoder can read the QR code.

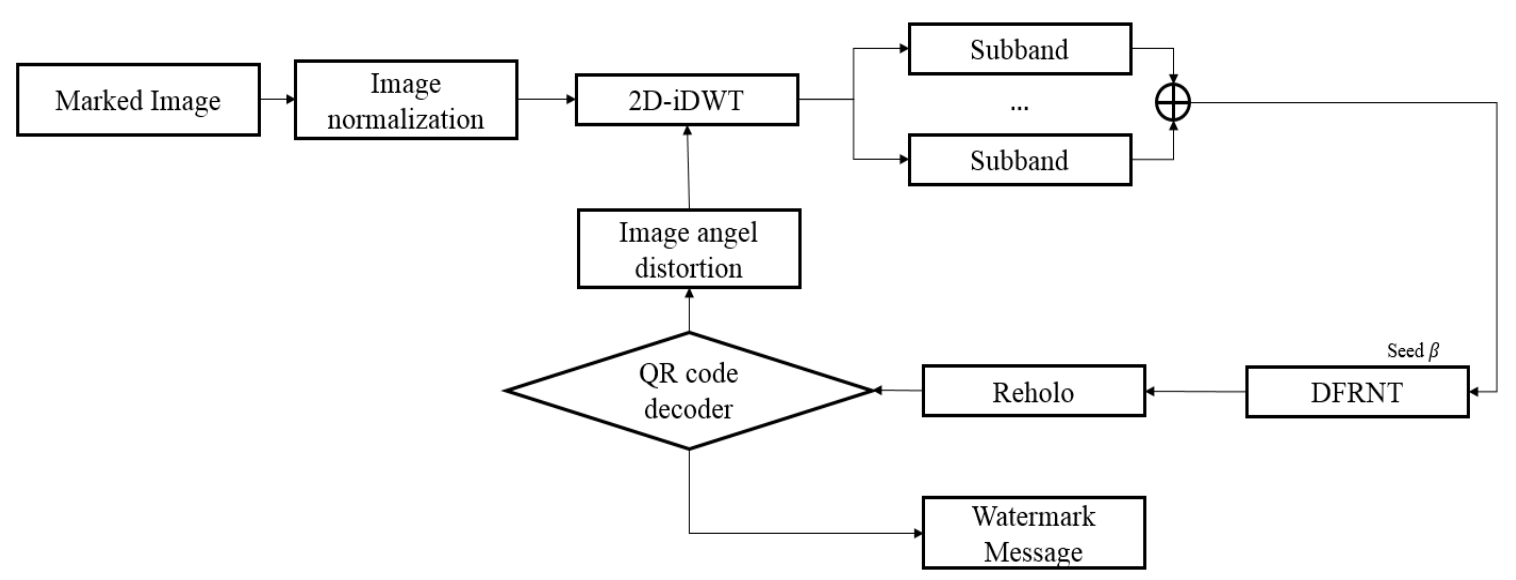

Figure 7. Watermark Extracting Process

\section{Experiments and Results}

In this paper, we used a QR code for the watermark message. QR codes consisted of black modules arranged in a square pattern on a white background. The performance of the proposed watermarking scheme was measured using the several city images with several difference size as shown in Figure 8.

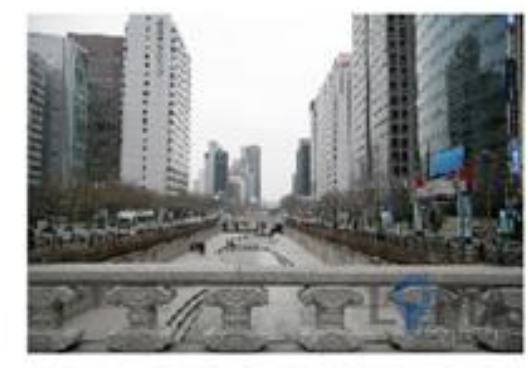

Width: 1,280 Height: 853

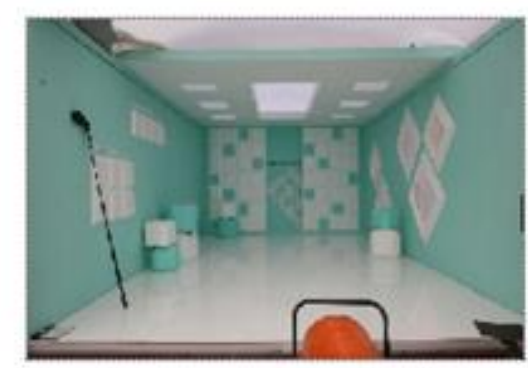

Width: 800 Height: 533 


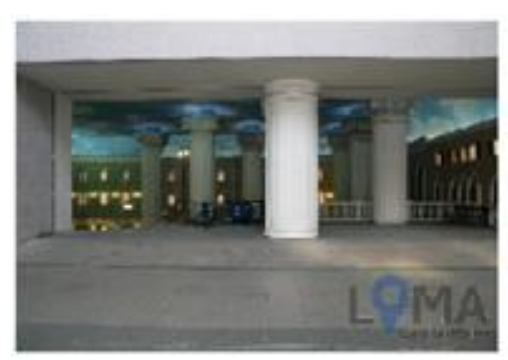

Width: 1,280 Height: 853

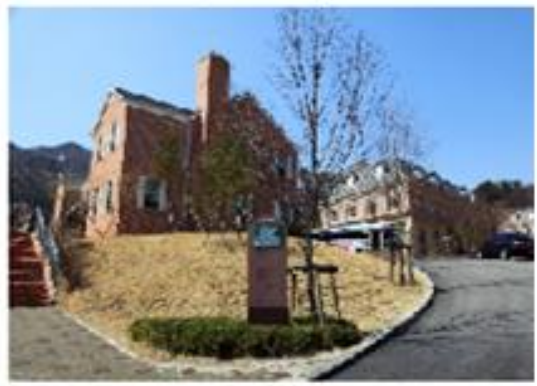

Width: 1,000 Height: 667

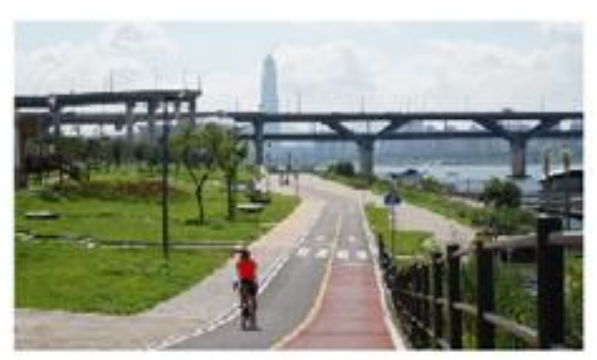

Width: 800 Height: 449

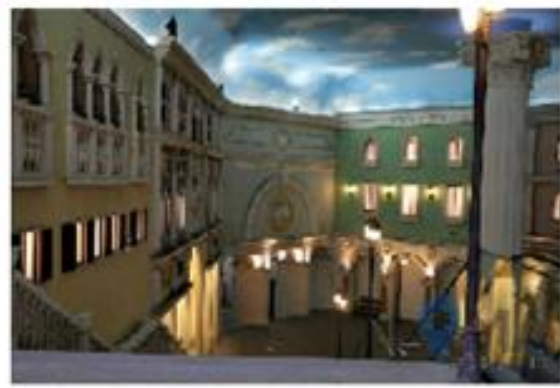

Width: 1,280 Height: 853

Figure 8. City Image

The size of the QR code was $21 \times 21$ and its payload was from 72 to 152 bits. It used Reed-Solomon error correction algorithm with four error correction levels. The higher the error correction level, the less storage capacity. According to the level, from 7 to $30 \%$ damaged QR Code can be restored.

For improving the accuracy of detecting, we fixed the center of image and swiveled that the pixels of image boundary move 1 notch at a time. In our experiments, the times of swiveling were 2 8 in clockwise and anticlockwise. Figure 9-B. shows the detected Rehologram matrixes. Only the fifth matrix is QR code and the decoder can read the message of watermark.

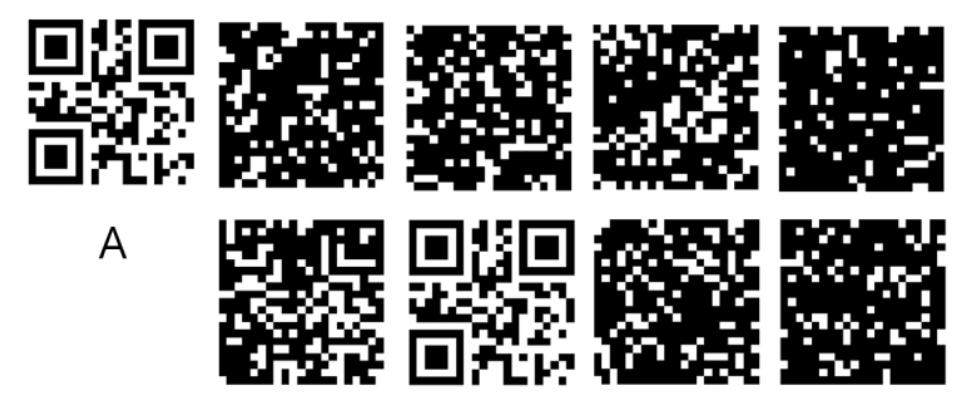

B

Figure 9. (A) The Embedded QR Code, (B) the Extracted QR Code

The watermark's invisibility is generally measured using the peak signal-to-noise ratio (PSNR) and the mean squared error (MSE):

$P S N R=20 \log _{10}(255 / M S E)$ 


$$
M S E=\sqrt{\frac{1}{M N} \sum_{x=1}^{M} \sum_{y=1}^{N}\left[f^{\omega}(x, y)-f(x, y)\right]^{2}}
$$

where $\mathrm{M}$ and $\mathrm{N}$ represent the width and height. Table 1 lists the PSNRs and BERs corresponding to the city images with several rotation attacks. Using the proposed method, we obtain BERs of $0-0.68 \%$.

\section{Table 1. PSNR and BER Values for Various City Images}

\begin{tabular}{|c|c|c|c|}
\hline Image & PSNR(dB) & Angle & BER (\%) \\
\hline City 1 & 45.6 & 0 & 0 \\
\hline & & 10 & 0 \\
\hline & & 20 & 0.22 \\
\hline City 2 & 47.6 & 0 & 0 \\
\hline & & 10 & 0 \\
\hline & & 20 & 0.68 \\
\hline City 3 & 48.6 & 0 & 0.22 \\
\hline & & 10 & 0.22 \\
\hline & & 20 & 0.68 \\
\hline City 4 & 50.3 & 0 & 0 \\
\hline & & 10 & 0.22 \\
\hline & & 20 & 0.22 \\
\hline
\end{tabular}

Experimental image is used as a "Photo Wonder" application for special effects. The application has been developed in Beijing Baidu Netcom Science \& Technology Company Limited. 


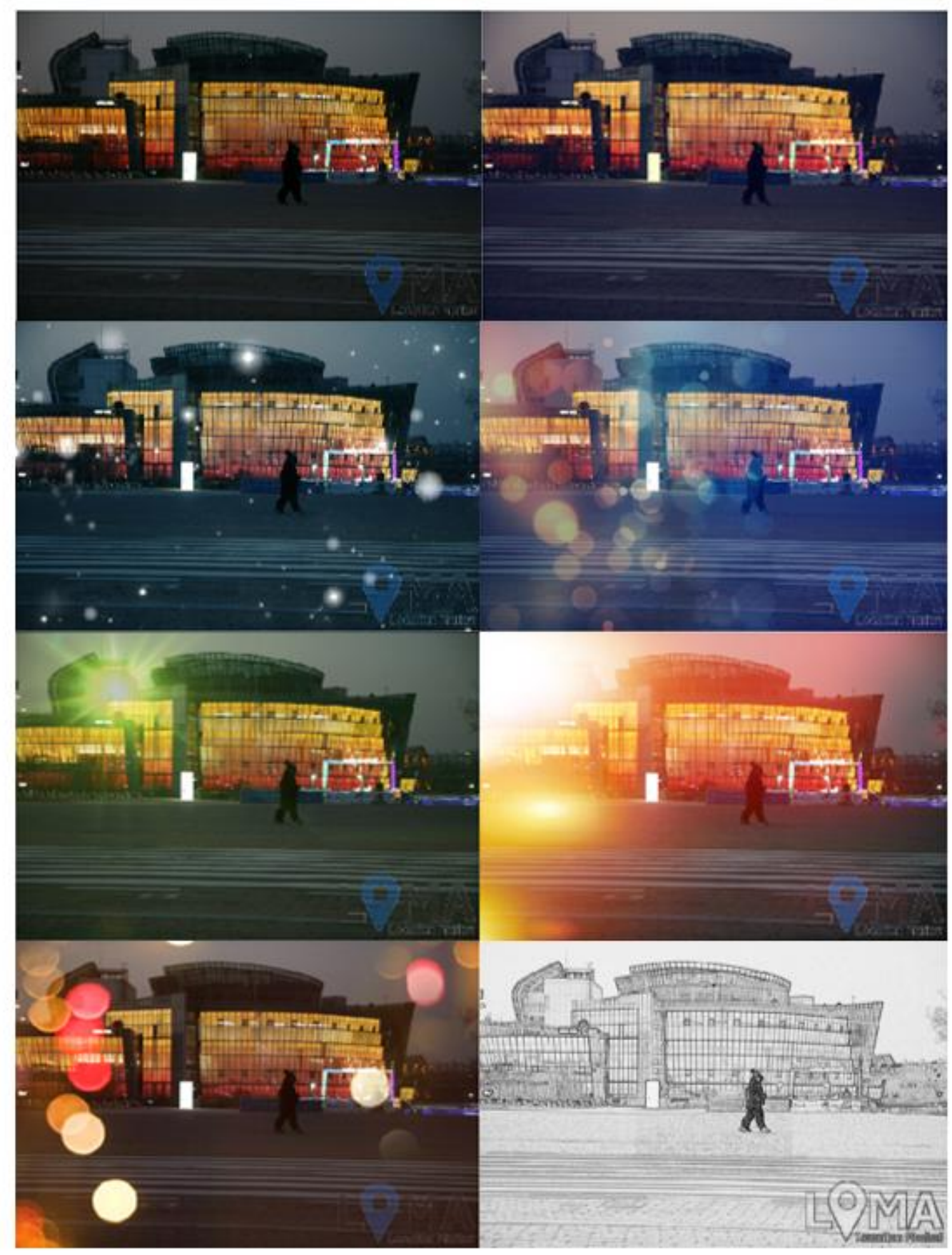

Figure 10. Effect Images

Figure 10. shows the original Image and several effects attack images. The effects are each LOMO Effect, Gorgeous Effect, Snow Effect, Warm Effect, Sunburst Effect, Neon Effect and Sketch Effect. 


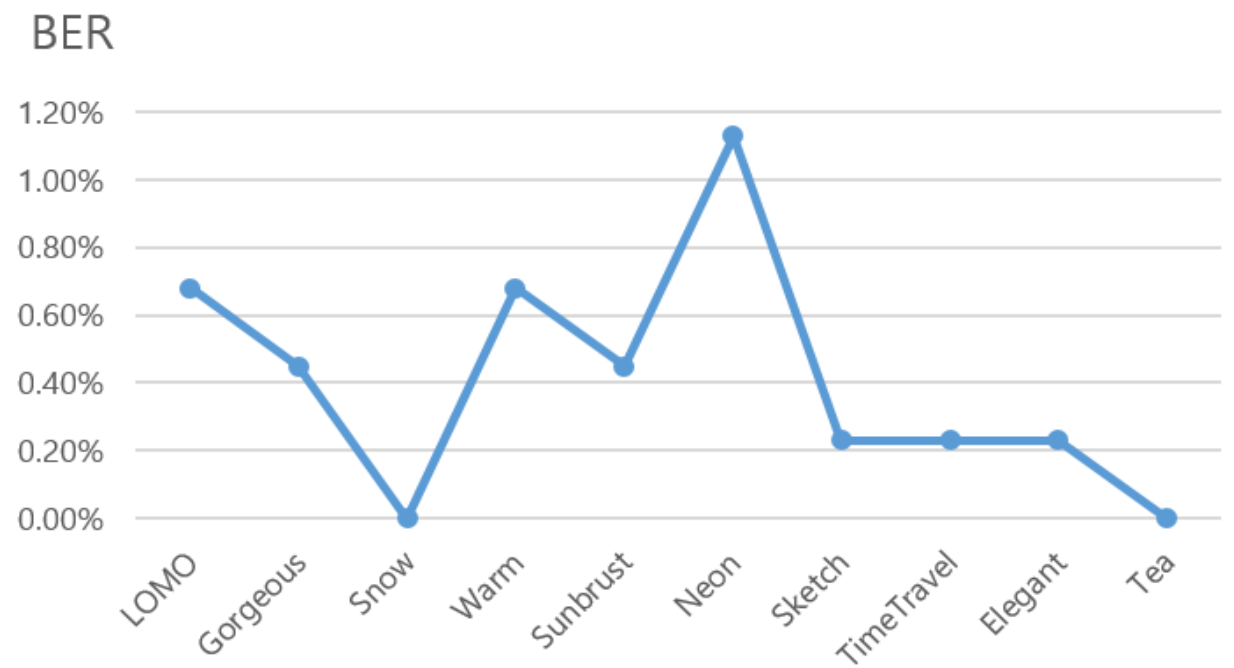

Figure 11. The Values of Bit Error Rate for Several Effect Attacks

Figure 11. shows the values of Bit Error Rate for several effect attacks. As shown in figure the BER values are obtain in $0 \%$ to $1.2 \%$. The BER of QR code is $0 \%$ in Snow Effect, Tea Effect attacks. After the Sketch Effect, Time Travel Effects and Tea Effect attacks, the values of BER are all $0.23 \%$. It means that the $\mathrm{QR}$ code has the 1pixcel point error in $21 \times 21$. According to the QR code attribution that can be restored from 7 to $30 \%$, the results with the values of BER are enough to restore the watermark information.

\section{Conclusion}

In this paper, we proposed a digital watermarking scheme for city image. It used image normalization that it efficient in rotation attack and produces better results. We distort the image to improve the accuracy. We fix the center of image and swivel that the pixels of image boundary move 1 notch at a time. We used random seed $\beta$ for security and embedded watermark after transforming by the iDWT. To evaluate the performance of the proposed method, watermark information was embedded in the wavelet-transformed domain. In rotation attack 0 20 degree, we detect the watermark with 0 0.68\% BER. We used the QR code as watermark which can restore the damaged QR code. Experimental results showed that the proposed method gives robustness under rotation attacks and special effect attacks.

\section{Acknowledgments}

This research is supported by Ministry of Culture, Sports and Tourism (MCST) and Korea Creative Content Agency (KOCCA) in the Culture Technology (CT) Research \& Development Program 2015.

\section{References}

[1] J. Kim, N. Kim, D. Lee, S. Park and S. Lee, "Watermarking two dimensional data object identifier for authenticated distribution of digital multimedia contents," Signal Processing: Image Communication 25, (2010), pp. 559-576.

[2] Y. Lee and J. Kim, "Robust Blind Watermarking scheme for Digital Images Based on Discrete Fractional Random Transform," Communications in Computer and Information Science 263, 139145 (2011).

[3] R. Jin and J. Kim, "Rotation-Invariant Image Watermarking Scheme Based on Radon Transform", Advanced Science and Technology Letters, vol. 120(DCA2015), pp753-758. 
[4] J. Nah, J. Kim and J. Kim, "Video Forensic Marking Algorithm Using Peak Position Modulation," Applied Mathematics \& Information Sciences (AMIS) 6(6S) (2012).

[5] Localized image watermarking based on feature points of scale-space representation," Pattern Recognition, Volume 37, Issue 7, July 2004, (2004), pp.1365-1375.

[6] F. A. P. Petitcolas, R. J. Anderson, and M. G. Kuhn, "Attacks on copyright marking systems," in Proc. Int. Workshop on Information Hiding, pp. 218-238, Springer-Verlag, 1998.

[7] M. Kutter, "Watermarking resisting to translation, rotation and scaling," Proc. SPIE 3528, (1998), pp. 423-431,

[8] S. Pereira and T. Pun, "Robust template matching for affine resistant image watermark," IEEE Trans. Image Process. vol. 9, no. 6, (2000), pp. 1123-1129.

[9] C. Lin and I. Cox, "Rotation, scale and translation resilient watermarking for images," IEEE Trans. Image Process. 10(5), pp. 767-782, 2001.

[10] J. Ruanaidh and T. Pun, "Rotation, scale and translation invariant spread spectrum digital image watermarking,” Signal Process. vol. 66, no. 3, (1998), pp. 303-317.

[11] P. Bas, J. Chassery, and B. Macq, "Geometrically invariant watermarking using feature points," IEEE Trans. Image Process. vol. 11, no. 9, (2002), pp. 1014-1028.

[12] M. Kutter, S. K. Bhattacharjee, and T. Ebrahimi, "Toward second generation watermarking schemes," in IEEE Int. Conf. on Image Processing,Vol. 1, pp. 320-323 , 1999.

[13] A. Nikolaidis and I. Pitas, "Region-based image watermarking," IEEE Trans. Image Process. vol. 10, no. 11, (2001), pp. 1726-1740.

[14] C. Tang and H. Hang, "A feature-based robust digital image watermarking scheme," IEEE Trans. Signal Process. vol.51, no. 4, (2003), pp. 950-959,

[15] D. Lowe, "Distinctive image features from scale-invariant keypoints," Int. J. Comput. Vis. vol. 60, no. 2, (2004), pp. 91-110.

[16] K. Mikolajczyk and C. Schmid, "Scale and affine invariant interest point detectors," Int. J. Comput. Vis. vol.60, no. 1, (2004), pp. 63-86.

[17] T. Tuytelaars and L. V. Gool, "Matching widely separated views based on affine invariant regions," Int. J. Comput. Vis. vol.59, no.1, (2004), pp. 61-85.

[18] S. Voloshynovskiy, A. Herrigel, N. Baumgartner, and T. Pun, "A stochastic approach to content adaptive digital image watermarking," in Proc. Int. Workshop on Information Hiding, Springer-Verlag, (1999), pp. 212-236.

[19] D. Gabor, “A New Microscope Principle,” Nature 161, (1948), pp.777.

[20] E. Leith, J. Upatnieks, "Reconstructed Wavefronts and Communication Theory," Journal of the Optical Society of America 52, (1962), pp. 1377.

[21] R. Jin and J. Kim, “A digital watermarking scheme using hologram quantization,” in Proc. International Conference on Signal Processing, Image Processing and Pattern Recognition 2012, vol. 342, November (2012), pp 39-46.

[22] Z. Liu, H. Zhao, S. Liu, "A discrete fractional random transform," Optical Communications vol. 255, no. 4-6, (2005), pp. 357-365.

[23] A. Paplinski, "Rotation invariant categorization of visual objects using Radon transform and selforganizing modules," in Lect. Notes in Comp. Sci. vol. 6444. Springer, (2010), pp. 360-366,

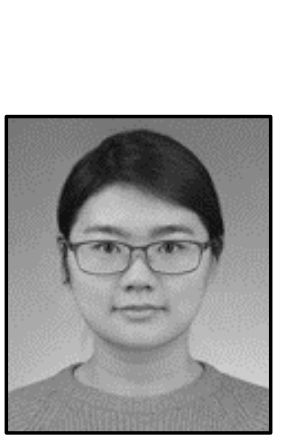

\section{Authors}

Ruichen Jin received her B.S. degree in Computer Science and Technology from YanBian University, China, in 2011. She is currently pursuing the Ph.D. degree in Copyright Protection, Sangmyung University, Korea. Her research interests are digital watermarking, multimedia forensics, digital signal processing, and information security.

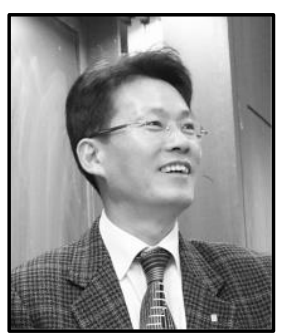

JongWeon Kim received the Ph.D. degree from University of Seoul, major in signal processing in 1995 . He is currently a professor of Dept. of Contents and Copyright at Sangmyung University in Korea. He has a lot of practical experiences in the digital signal processing and copyright protection technology in the institutional, the industrial, and academic environments. His research interests are in the areas of copyright protection technology, digital rights management, digital watermarking, and digital forensic marking. 
International Journal of Security and Its Applications Vol.10, No.1 (2016) 